University of Nebraska - Lincoln

DigitalCommons@University of Nebraska - Lincoln

Papers in Microbiology

Papers in the Biological Sciences

October 2006

\title{
Pichia pastoris fermentation optimization: energy state and testing a growth-associated model
}

\author{
Bradley A. Plantz \\ University of Nebraska-Lincoln, bplantz2@unl.edu \\ Jayanta Sinha \\ University of Nebraska-Lincoln \\ Lorelie Villarete \\ Pepgen, Inc. \\ Kenneth W. Nickerson \\ University of Nebraska-Lincoln, knickerson1@unl.edu \\ Vicki Schlegel \\ University of Nebraska-Lincoln, vschlegel3@unl.edu
}

Follow this and additional works at: https://digitalcommons.unl.edu/bioscimicro

Part of the Microbiology Commons

Plantz, Bradley A.; Sinha, Jayanta; Villarete, Lorelie; Nickerson, Kenneth W.; and Schlegel, Vicki, "Pichia pastoris fermentation optimization: energy state and testing a growth-associated model " (2006). Papers in Microbiology. 74.

https://digitalcommons.unl.edu/bioscimicro/74

This Article is brought to you for free and open access by the Papers in the Biological Sciences at DigitalCommons@University of Nebraska - Lincoln. It has been accepted for inclusion in Papers in Microbiology by an authorized administrator of DigitalCommons@University of Nebraska - Lincoln. 


\title{
Pichia pastoris fermentation optimization: energy state and testing a growth-associated model
}

\author{
Bradley A. Plantz, ${ }^{1}$ Jayanta Sinha, ${ }^{2}$ Lorelie Villarete, ${ }^{3}$ Kenneth W. Nickerson, ${ }^{1}$ \\ and Vicki L. Schlegel ${ }^{4 *}$ \\ ${ }^{1}$ School of Biological Sciences, Beadle Center, University of Nebraska-Lincoln, Lincoln, NE 68583-0666, USA \\ 2 Department of Chemical Engineering, Othmer Hall, University of Nebraska-Lincoln, Lincoln, NE 68583-0668, USA \\ ${ }^{3}$ Pepgen, Inc., 1265 Harper Bay Parkway, Alameda, CA 94502, USA \\ ${ }^{4}$ Department of Food Science and Technology, Filley Hall, University of Nebraska-Lincoln, Lincoln, NE 68583-0919, USA \\ * Corresponding author: e-mail: vlschleg@unlnotes.unl.edu Tel. 402 472-4694, Fax 402 472-1693
}

\begin{abstract}
A growth-associated model was applied to the production of recombinant ovine interferon- $\tau$ (rOvIFN- $\tau$ ) with Pichia pastoris for the purpose of manufacturing preclinical and clinical active material. This model predicts that product yields will be the greatest when the specific growth of the culture is maintained at a steady and optimal rate. However, rOvIFN- $\tau$ yields did not meet the expected linear model but most closely corresponded to a polynomial relationship. After transitioning from glycerol to methanol, product accumulated for $31-45 \mathrm{~h}$, and then the yield decreased. This production shift, which has been termed decoupling, was clearly related to time on methanol and not culture density. It was determined that a correlation exists between the decoupling point and a drop in energy state of the cell when expressing $\beta$-galactosidase. By assigning decoupling as a constraint that limits productivity and by reformulating the growth medium, the time prior to decoupling increased to $46.8 \pm 2.4 \mathrm{~h}$, product yield improved for rOvIFN- $\tau$ from 203 to $337 \mathrm{mg} \mathrm{l}^{-1}$, and the coefficient of variation for yield decreased from 67.9 to $23.3 \%$. A robust and stable fermentation process was realized, resulting in a $210 \%$
\end{abstract}

\section{Introduction}

Production of more than 400 recombinant proteins have been reported with the mut ${ }^{+}$strain of the methylotrophic yeast Pichia pastoris by linkage to the alcohol oxidase 1 (AOX1) promoter and by applying high cell density fedbatch fermentations (Lin-Cereghino et al. 2002; Tschopp et al. 1987; Cregg et al. 1985). The most widely utilized fermentation approach at both the bench-top and commercial scale consists of a four-step fed-batch process (Stratton et al. 1998). During the first step, cell mass increases by allowing the culture to grow on defined medium with glycerol as the carbon source. A glycerol fed-batch phase is then initiated to further increase the culture mass and to begin transitioning the cells from glycerol to methanol, the inducing carbon source. Methanol is delivered to the culture at low levels in the transition phase, whereas the glycerol feed is either discontinued or continued at a nonrepressive rate. The fourth phase, production, typically requires a continuous feed of methanol. While fermentation methods have been developed with glycerol/methanol mixed feeds (Katakura et al. 1998; Zhang et al. 2003), the typical fermentation strategy uses methanol as the sole source for carbon and energy (Zhang et al. 2000a).

Because methanol is inhibitory at levels greater than 4$6 \mathrm{mg} \mathrm{l}^{-1}$, feed rates must be controlled during production (Katakura et al. 1998; Zhang et al. 2000b). The classical protocol incorporates a dissolved oxygen spike to control residual methanol, while the methanol feed rate is arbitrarily increased as culture density increases (Stratton et al. 1998). Van Heeke et al. (1996) was the first group to develop a fermentation process to produce recombinant ovine interferon- $\tau$ (rOvIFN- $\tau$ ) based on this method. However, when applied to fermentations completed in our laboratory, rOvINF- $\tau$ yields were insufficient to meet the needs of the planned clinical trials, and run-to-run variability was so ex- 
cessive that downstream processing parameters were severely impacted. As such, the process as originally developed by Van Heeke et al. (1996) could not be validated as required by the US Food and Drug Administration (CFRFDA) for biopharmaceuticals destined for human health care purposes.

Higher product yields for many recombinant proteins have resulted by developing a model for methanol utilization and then using that model to predict and control specific growth rates (Kobayashi et al. 2000; Zhang et al. 2000b; d'Anjou and Daugulis 2001). When this fermentation strategy was applied to the rOvIFN- $\tau$ process, maximal yields occurred at an optimal and constant culture growth rate of approximately 0.025 per hour (Sinha et al. 2003). Yet, product yield maximums were variable and shifted from accumulation to loss as early as $28 \mathrm{~h}$ for some processes and well past $45 \mathrm{~h}$ for other fermentations. Simply decreasing the production time could not remedy this problem because productivity would also decrease due to the broad range of peak production times. It was thus hypothesized that the expression of recombinant protein is regulated downstream from gene transcription. The prevailing tenet is that the AOX1 promoter is derepressed when methanol is present, and both AOX1 and the recombinant gene are constitutively expressed. This precept may be false because studies with Escherichia coli (Wood and Peretti 1990) and the methylotrophic yeast Hansenula polymorpha (Velkov et al. 1999) have shown that recombinant protein expression is limited somewhere downstream from transcription. If $P$. pastoris expression systems respond to recombinant protein synthesis in a similar manner, then strain and process improvements must be directed toward minimizing the metabolic limitation. This work builds upon the previously cited studies by evaluating the yield profiles of rOvIFN- $\tau$, and then of $\beta$-galactosidase, to determine if a shift in the cell's physiology correlates to the shift in the yield profile. More specifically, by monitoring adenylate energy charge (AEC) of the culture during the production phase, it was determined that the decoupling point correlated to a drop in the cells' energy state during the production of $\beta$-galactosidase. The high cell density fermentation process was also evaluated to access if a nutrient or metabolic intermediate might be limiting. The information generated from these studies was then used to increase product yields and to stabilize run-torun variability of the rOvIFN- $\tau$ fermentation.

\section{Materials and methods}

Pichia pastoris expression systems

A research seed bank was prepared from a single cell isolate of $P$. pastoris strain X-33 mut ${ }^{+}$, (Invitrogen, Carlsbad, $\mathrm{CA})$ constructed with two chromosomal inserts under the control of the AOX1 promoter for expressing rOvIFN- $\tau$ (Pepgen, Inc., Alameda, CA). The stock culture, inoculum, and the bench-top fermentations were screened for nonhost-viable contaminants (Plantz et al. 2003). P. pastoris strain GS-115 his $^{-}$(Invitrogen) was transformed with the plasmid pSAOH5 (James Cregg, Keck Graduate Institute, Claremont, CA) containing the lacZ construct to produce a mut $^{+}$, his ${ }^{+}$phenotype (Tschopp et al. 1987). Stock cultures designated BP5 were derived from a single isolate that was screened for genetic stability and production of $\beta$-galactosidase.

\section{Chemicals, reagents, and growth medium}

All chemicals used for culture growth were of suitable commercially available grades. Inoculums for bench-top fermentation were prepared with buffered minimal glycerol (BMGY) medium that consisted of (in grams per liter) yeast extract, 12.6; soytone, 10; yeast nitrogen base without amino acids, 13.4; and buffered with $100 \mathrm{mM}$ potassium phosphate ( $\mathrm{pH}$ 6.0). Inoculums for shake-flask studies were grown in yeast extract peptone glycerol (YPG) containing (in grams per liter) yeast extract, 5; peptone, 10; and glycerol, 10; adjusted to $\mathrm{pH}$ 5.5. Unless otherwise specified, the bench-top fermentation medium was FM22, which was composed of (in grams per liter) $\mathrm{KH}_{2} \mathrm{PO}_{4}, 42.9 ;\left(\mathrm{NH}_{4}\right)_{2} \mathrm{SO}_{4}, 5 ; \mathrm{CaSO}_{4} \cdot 2 \mathrm{H}_{2} \mathrm{O}, 1.0 ; \mathrm{K}_{2} \mathrm{SO}_{4}$, 14.3; $\mathrm{MgSO}_{4} \cdot 7 \mathrm{H}_{2} \mathrm{O}, 11.7$; and glycerol, 40 (Stratton et al. 1998). Pichia trace minerals 4 (PTM4) salt solution was prepared with (in grams per liter) $\mathrm{CuSO}_{4} \cdot 5 \mathrm{H}_{2} \mathrm{O}, 2.0 ; \mathrm{NaI}$, $0.08 ; \mathrm{MnSO}_{4} \cdot \mathrm{H}_{2} \mathrm{O}, 3.0 ; \mathrm{Na}_{2} \mathrm{MoO}_{4} \cdot 2 \mathrm{H}_{2} \mathrm{O}, 0.2 ; \mathrm{H}_{3} \mathrm{BO}_{3}, 0.02$; $\mathrm{CaSO}_{4} \cdot 2 \mathrm{H}_{2} \mathrm{O}, 0.5 ; \mathrm{CoCl}_{2}, 0.5 ; \mathrm{ZnCl}_{2}, 7 ; \mathrm{FeSO}_{4} \cdot 7 \mathrm{H}_{2} \mathrm{O}, 22$; biotin, 0.2 ; and $1 \mathrm{ml}$ of concentrated $\mathrm{H}_{2} \mathrm{SO}_{4}$ (Stratton et al. 1998). Medium was formulated in 10-1 batches to reduce between batch differences. Sterile filtered methanol supplemented with $2 \mathrm{ml} / 1$ PTM4 salts was prepared for the production phase. The buffer used for washing cultures was composed of (in grams per liter) $\mathrm{KCl}$, 9; sodium citrate, 2.9; and 2-(N-morpholino) ethanesulfonic acid (MES), 2.0 (adjusted to $\mathrm{pH}$ 5.5).

\section{rOvIFN- $\tau$ fermentation production conditions}

Inoculums were prepared by transferring $500 \mu \mathrm{l}$ of thawed stock culture to $500 \mathrm{ml}$ of BMGY medium contained within stopper baffled shake flasks. The culture was incubated with shaking at $28 \pm 2{ }^{\circ} \mathrm{C}$ for $36-48 \mathrm{~h}$. FM22 medium (2 1) was transferred to a fermentation vessel (4-1 working volume) that was steam-sterilized. The medium was supplemented with $2.0 \mathrm{ml} / 1$ of filter-sterilized PTM4 solution and adjusted to $\mathrm{pH} 5.0$ with saturated aqueous ammonium hydroxide. The shake-flask inoculums were transferred to each fermentation vessel in 120-ml aliquots. Temperature, $\mathrm{pH}$, and relative dissolved oxygen (DO) were monitored and maintained by the fermentation unit (BioFlo III and BioFlo 3000; New Brunswick Scientific Co., Edison, NJ). The $\mathrm{pH}$ was adjusted with saturated ammonium hydroxide as needed. Dissolved oxygen was controlled by increasing the agitation rate to $800 \mathrm{rpm}$ followed by supplementation with $\mathrm{O}_{2}$, and the temperature was maintained at $30^{\circ} \mathrm{C}$. 
During the first fermentation phase, the cells were allowed to grow until the batch glycerol was exhausted, as indicated by a DO greater than $10 \%$ of the set point. The glycerol fed-batch phase consisted of feeding $50 \%$ (wt/wt) glycerol into the fermentor at a rate of $20 \mathrm{~g} \mathrm{l}^{-1} \mathrm{~h}^{-1}\left(10 \mathrm{~g} \mathrm{l}^{-1}\right.$ $\mathrm{h}^{-1}$ glycerol) for 1 hand ramping down the feed rate to $0 \mathrm{~g}$ $\mathrm{l}^{-1} \mathrm{~h}^{-1}$ over the next $3 \mathrm{~h}$. The glycerol feed rate was controlled by a time-based program included in the BioCommand 32 software. The $\mathrm{pH}$ was adjusted to 6.0 with ammonium hydroxide during the 4-h glycerol fed-batch phase. Transition to methanol was accomplished by adding 1.5 $\mathrm{g}^{-1}$ methanol to the fermentor at the start of the glycerol ramp down period. Conversion to growth on methanol was confirmed by monitoring residual methanol levels in the offgas. The transition period was considered complete when the detection limit of the sensor (PTI Instruments, Kathleen, GA) was reached at approximately $0.01 \mathrm{~g} \mathrm{l}^{-1}$. The feed rate of the methanol was controlled as described by Zhang et al. (2002) and applied to rOvIFN- $\tau$ production as described by Sinha et al. (2003). Briefly, methanol control equations were programmed with New Brunswick Scientific BioCommand32 supervisory software. Methanol consumption was controlled by a closed-loop system consisting of a variable-speed Watson-Marlow feed pump (model $101 \mathrm{U} / \mathrm{R}$, England), a Mettler Toledo balance for weighing methanol (model PG12001-s, Switzerland) and a controller interface with the BioCommand32 software. Temperature, $\mathrm{pH}$, and DO were maintained at set points of $30^{\circ} \mathrm{C}, 6.0$, and $40 \%$, respectively, throughout the production phase.

\section{Fermentation analysis}

Culture growth rate and cell mass were determined by collecting samples at regular intervals and centrifuging the suspension in preweighed conical tubes. After decanting the supernatant, duplicate samples of cell mass were measured gravimetrically and recorded as wet cell weight (WCW) per liter. The volume of cell broth was monitored by shutting off the agitator and measuring the settled suspension against calibrated $0.25-1$ graduations marked on the fermentor vessel.

The specific growth rate $(\mu)$ was determined by integrating over a time period of $t_{0}$ to $t$ according to the equation for growth in a fed-batch system,

$$
d(X V)=\mu X V d t
$$

producing an exponential equation of the type $y=a e^{\mu t}$. The specific rate of product formation, $\rho$ (mg rOvIFN- $\tau$ g $\mathrm{WCW}^{-1} \mathrm{~h}^{-1}$ ), was obtained from the slope of the linear plot of total yield rOvIFN- $\tau(J)$ vs $\left(X V-X_{0} V_{0}\right) / \mu$, where $X$ and $V$, and $X_{0}$ and $V_{0}$, are the density of cells and volume of the fermentor at the end and initiation of the methanol feed profile, respectively (Zhang et al. 2000b; Sinha et al. 2003).

Recombinant ovine interferon tau yields were measured by high-performance liquid chromatography (HPLC). The supernatants collected from the WCW measurements were filtered through a $0.45-\mu \mathrm{m}$ syringe filter and stored frozen at $-20^{\circ} \mathrm{C}$ until analyzed. Reverse-phase HPLC was performed with a Vydac C4 column $(4.6 \mathrm{~mm} \times 150 \mathrm{~cm})(\mathrm{Co}-$ lumbia, MD), a 484 Waters UV-Vis detector, and a Waters 626 LC pump (Milford, MA). Mobile phase A was prepared with $90 \%$ water, $0.1 \%$ trifluoroacetic acid, and $10 \%$ acetonitrile, whereas mobile phase $\mathrm{B}$ was prepared with $10 \%$ water, $90 \%$ acetonitrile, and $0.1 \%$ trifluoroacetic acid. Samples were placed in a Waters 717 autosampler and maintained at $4^{\circ} \mathrm{C}$ prior to injection. After a sample injection of $100 \mu \mathrm{l}$, the column was flushed for $5 \mathrm{~min}$ with

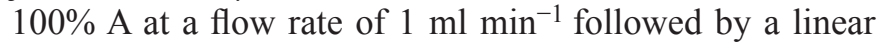
gradient to $100 \%$ B over 30 min that was held at $100 \%$ B for another $5 \mathrm{~min}$. The rOvIFN- $\tau$ peak eluted at a retention time of $22-23 \mathrm{~min}$, as detected at wavelength $280 \mathrm{~nm}$. A well-characterized rOvIFN- $\tau$ standard was used to generate a calibration curve.

\section{Analysis of adenylate energy charge}

BP5 cultures were first grown on FM22 with 4\% glycerol supplemented with PTM4 salt solution. A washed suspension of cells was transferred to $0.1 \times$ FM22/PTM4 defined medium at a targeted concentration of $5 \mathrm{OD}_{600} \mathrm{ml}^{-1}$. After adding $0.01 \%$ methanol, the cells were transferred to a $250-\mathrm{ml}$ baffled shake flask in 50-ml aliquots. Methanol was added intermittently to sustain growth. At the indicated time points, 2-ml samples were removed and transferred into $4 \mathrm{ml}$ of boiling water to quench metabolism and lyse the cells (Babul et al. 1993). A maximum of $1 \mathrm{~min}$ elapsed between collecting the sample and quenching. The samples were boiled for $5 \mathrm{~min}$, rapidly cooled on ice, centrifuged, and supernatants were evaporated to dryness with a centrivap. Like samples were pooled into $500 \mu \mathrm{l}$ of nanopure water and stored frozen at $-20^{\circ} \mathrm{C}$. Molar quantities of each adenylate were determined by the Waters HPLC instrumentation system described above. Adenylates were identified by matching the retention times and evaluating peak heights between the preset scan limits and the expected absorption maximum of $254 \mathrm{~nm}$. Mobile phase A consisted of $100 \mathrm{mM}$ potassium phosphate and $8 \mathrm{mM}$ tetrabutyl ammonium phosphate, whereas mobile phase $\mathrm{B}$ was prepared as $30 \%$ methanol, $100 \mathrm{mM}$ potassium phosphate, and $8 \mathrm{mM}$ tetrabutyl ammonium phosphate. A Vydac $(4.6 \times 250 \mathrm{~mm})$ C-18 column was equilibrated with mobile phase A for 15 min between each injection at a flow rate of $1 \mathrm{ml} \mathrm{min}^{-1}$. The gradient run conditions were $0 \mathrm{~min}, 10 \% \mathrm{~B} ; 0-10 \mathrm{~min}, 10$ $23 \% \mathrm{~B} ; 10-15 \mathrm{~min}, 23 \% \mathrm{~B}$; $15-16 \mathrm{~min}, 23-50 \% \mathrm{~B} ; 16-26$ $\min , 50 \% \mathrm{~B} ; 26-27 \mathrm{~min}, 50-75 \% \mathrm{~B} ; 27-32 \mathrm{~min}, 75 \% \mathrm{~B}$; $32-33 \mathrm{~min}, 75-100 \% \mathrm{~B} ; 33-43 \mathrm{~min}, 100 \% \mathrm{~B}$. The stability of the samples stored in the auto-injector was confirmed by comparing chromatograms of a sample injected at the start of each series and at the end of that series. No changes in retention times or peak profiles were observed. The AEC was calculated according to Atkinson and Walton (1967). 
Figure 1. Culture growth and $\mathbf{2 0 0 0}$ product yield data for $P$. pastoris X-33 mut $^{+}$expressing rOvIFN- $\tau$. Data are plotted at each time point as the total yield for either cell mass $(\boldsymbol{\Delta})$ or rOvIFN- $\tau(\square)$. The dashed line shows the regression curve generated from the aggregate cell mass data

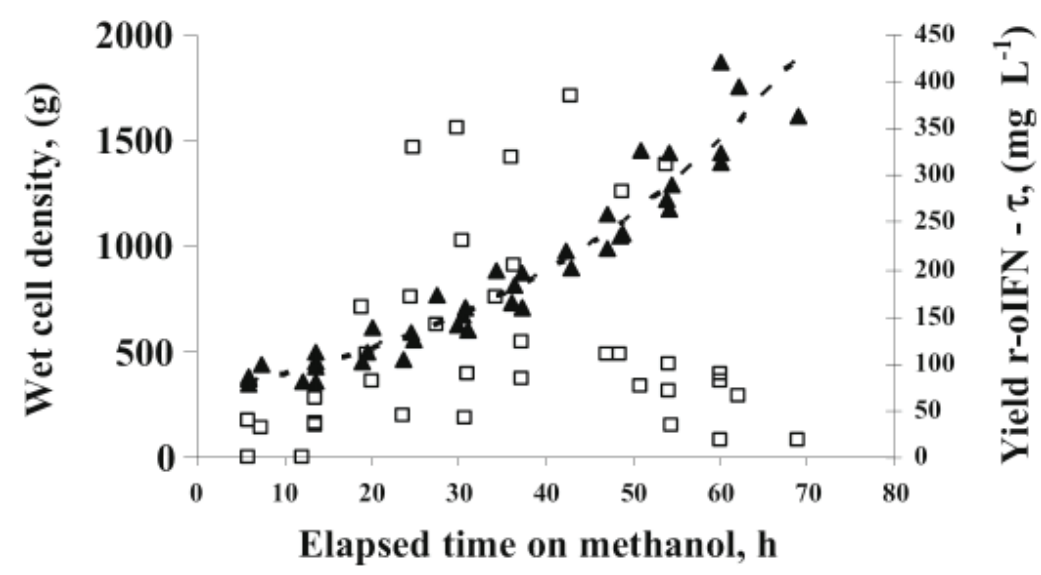

were not maintained at the designated set points, the experiment was repeated.

To compare the experimental product yields with those predicted by the growth-associated model, the yield of rOvIFN- $\tau$ vs the change in cell mass normalized to $\mu$ was plotted (Figure 2) as described by Zhang et al. (2000b) and Sinha et al. (2003). Although a linear relationship was expected (Figure 2, soft dash), the data most closely fit a second-order polynomial relationship (Figure 2, solid curve). The maximum mean total product yield of $447 \pm 154 \mathrm{mg}$ was reached when the total culture density was $805 \pm 221$ g WCW, which occurred $38.5 \pm 6.7 \mathrm{~h}$ after the start of the methanol profile. Considering that the total cellular capacity of the vessel was $1,800 \mathrm{~g} \mathrm{WCW}$ in 41 of broth, the maximum yield was reached when the productive capacity of the vessel was at $45 \%$ of the maximum. However, the rOvIFN- $\tau$ yield data corresponded to a growth-associated model from the point of methanol adaptation to the yield maxima (Figure 2, hard dash line). For this segment of the production curve, $\rho$ was $0.025 \pm 0.014$, with $\mathrm{R}^{2}=0.58$. The deviation from the growth-associated model occurred after the decoupling point.

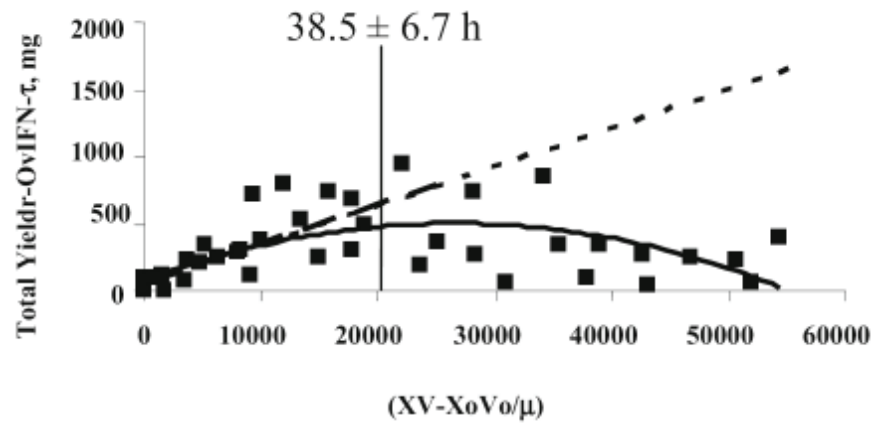

Figure 2. Yield model for rOvIFN- $\tau$. Yield of product ( $\mathbf{\square}$ ) from five replicate baseline fermentations plotted as a function of changing culture density normalized to specific growth rate $[(\mathrm{XV}-\mathrm{X} 0 \mathrm{~V} 0) / \mu]$. The rate of total vessel product accumulation (mg $\operatorname{rOvIFN}-\tau \times \mathrm{h}^{-1}$ ) is calculated from the slope of the regression lines. The solid curve is the best fit for the data. The large hash (-) is the regression line for the data prior to decoupling. The dashed extension (--) shows the predicted yield if $\rho$ had fit the growth-associated model over the entire methanol feed profile. 

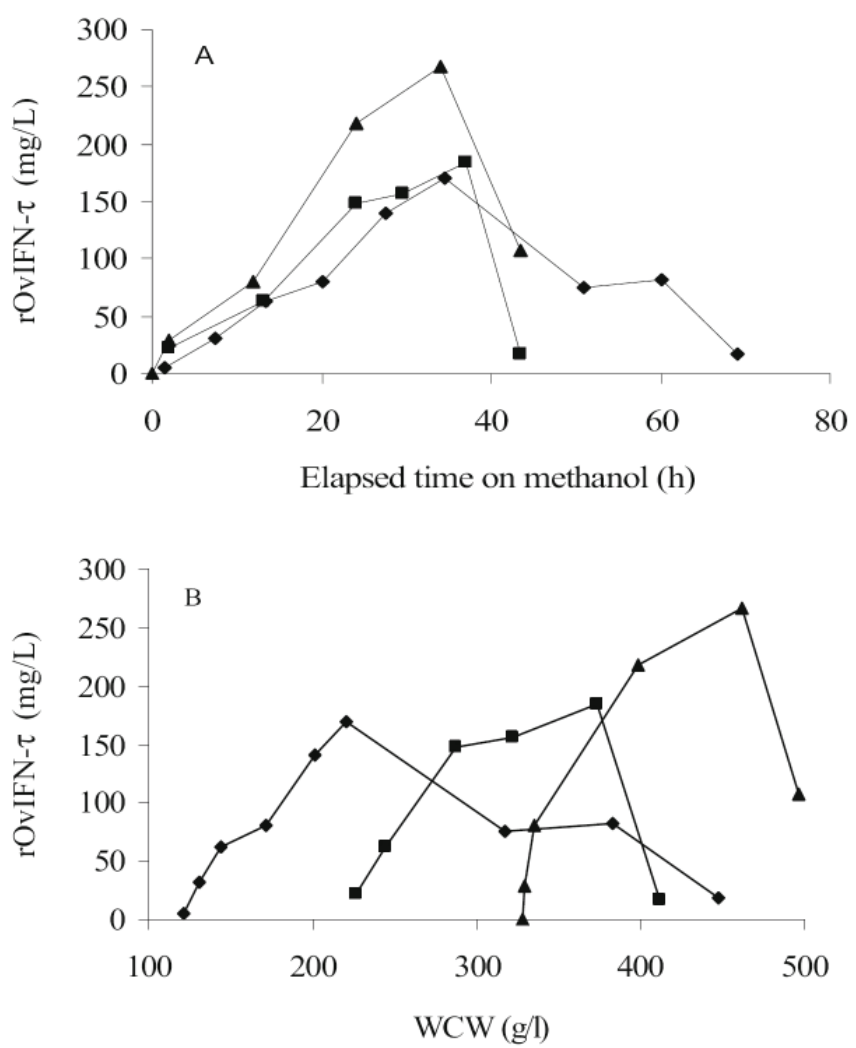

Figure 3. The relationship between the feed time of methanol (a) and culture density (b) on the yield of rOvIFN- $\tau$. Three otherwise identical fermentations were transitioned from glycerol to methanol at low $(\diamond)$, medium ( $\mathbf{\square})$ or high $(\boldsymbol{\Delta})$ culture densities relative to one another. The decoupling point occurs at nearly the same point after the transition from glycerol to methanol, while the culture density appeared to have no effect on the decoupling point.

\section{Influence of time and culture density on the decoupling point}

To confirm that time on methanol, and not culture density, was influencing the decoupling point, three fermentations were transitioned from glycerol to methanol at low, medium, or high culture density relative to each other. Prod- uct yields were compared as either a function of time or culture density (Figure 3 ). With respect to time after transition to growth on methanol (Figure 3a), the yield decoupled from growth at $34.5,37.0$, and $34.0 \mathrm{~h}$ [mean $=35.2$ $\mathrm{h}$; standard deviation $(\mathrm{SD})=1.6 \mathrm{~h}$ ] for the low, medium, and high cell density fermentations, respectively (Figure $3 b$ ). Relative to culture density (Figure $3 \mathrm{~b}$ ), product yield decoupled from growth at $221 \mathrm{~g} \mathrm{l}^{-1} \mathrm{WCW}$ (low culture density), $373 \mathrm{~g}^{-1} \mathrm{WCW}$ (medium culture density), and $462 \mathrm{~g} \mathrm{l}^{-1}$ WCW (high culture density) (mean $=352 \mathrm{~h}$; SD $=122)$. The coefficient of variation $(\mathrm{CV})$ for these two data series were $5.0 \%$ and $34.6 \%$, which confirms that decoupling is the outcome of time on methanol and not culture density.

\section{Changes in energy state}

The transformed GS115-mut ${ }^{+}$strain, designated BP5, was used to monitor the energy state of the cell because a large number of samples could be rapidly assayed for $\beta$-galactosidase. Compared to quasi-steady-state fed-batch fermentations, shake flasks are poorly controlled with respect to growth rate and $\mathrm{pH}$. Still, a constant growth rate of 0.051 per hour was maintained to the expected maximum sustainable culture density, indicating that methanol was not limited during exponential growth. The yield of $\beta$-galactosidase per cell reached a maximum between 12 and 24 $\mathrm{h}$, although culture growth continued to $48.5 \mathrm{~h}$ (Figure 4). Just after the glycerol-to-methanol transition, but before any significant growth or $\beta$-galactosidase had been detected, the AEC of the culture was $0.75 \pm 0.02$ (Figure 5). The AEC stabilized at approximately 0.70 for the next 12 $\mathrm{h}$ and then dropped to $0.60 \pm 0.05$, a significantly lower value compared to the initial AEC value $(p=0.005)$. The AEC decreased primarily because adenosine triphosphate (ATP) decreased and adenosine diphosphate (ADP) increased, whereas adenosine monophosphate (AMP) remained fairly constant to the 25-h sample (Figure 6d). The ADP and ATP peaks could not be resolved at the 25-h sample and thereafter because of additional peaks present in the chromatogram.
Figure 4. Growth curve and yield for $\beta$ galactosidase. The data were obtained from four replicate flasks at each time point, and the error bars show 1 standard deviation from the mean $(n=4)$. After the adaptation phase was completed, the culture grew at a specific growth rate of 0.0511 per hour $(\boldsymbol{\Delta})$. The yield of $\beta$-galactosidase (-m-) is shown as a function of the optical density.

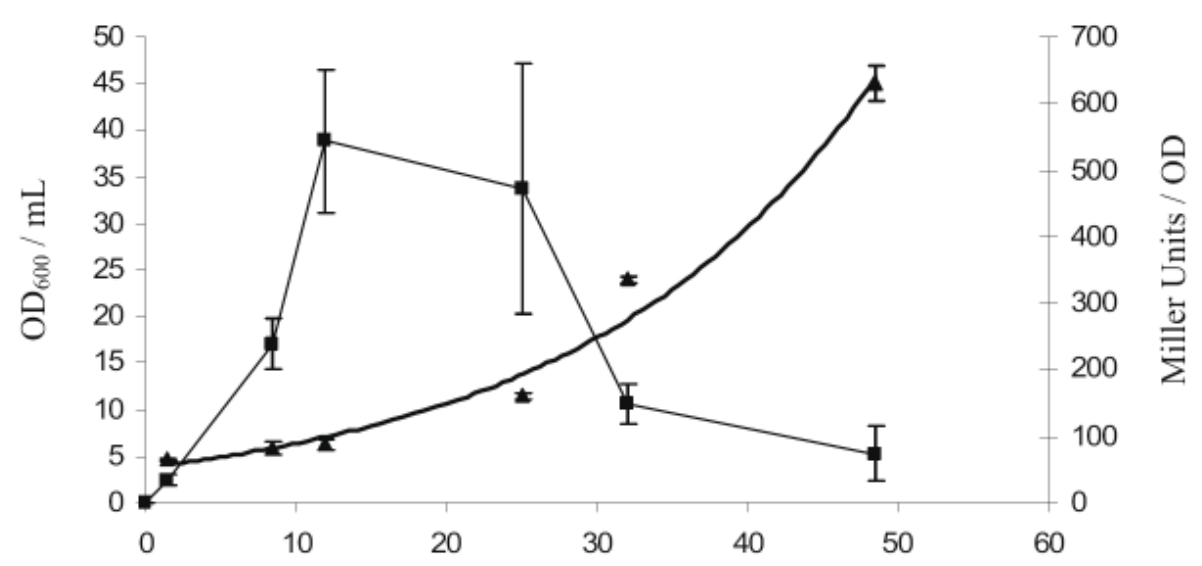

Elapsed time on methanol (h) 


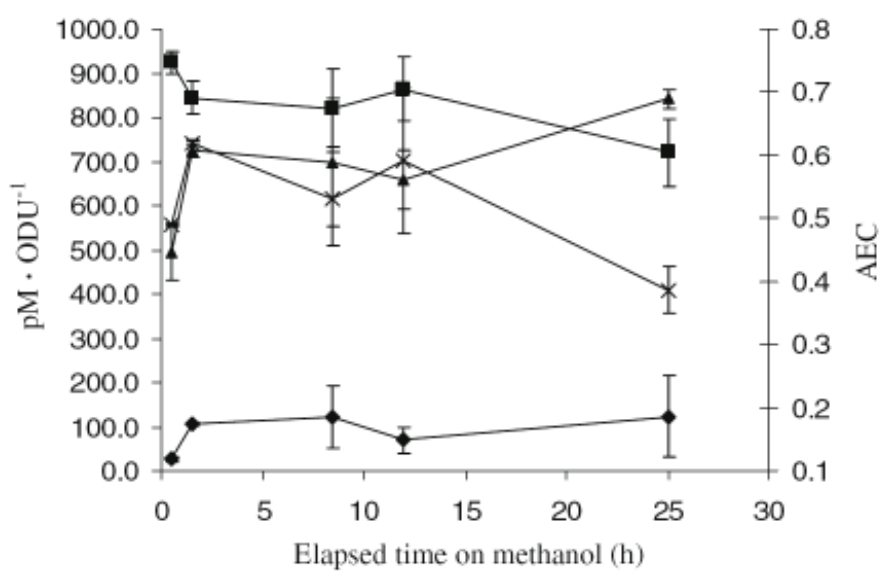

Figure 5. Changes in the energy state during expression of $\beta$-galactosidase. Changes in the energy state of the cell are reflected in the adenylate pool size $(\diamond$ AMP, $\boldsymbol{\Delta}$ ADP, $\times$ ATP) and adenylate energy charge (घ). Error bars show 1 standard deviation from the mean collect from four separate, replicate flasks.

\section{Improved basal salt medium}

Prior to developing the culture growth and product yield model for rOvIFN- $\tau$ (Sinha et al. 2003), studies were completed with basal salt metal (BSM) (Stratton et al. 1998) and Pichia trace metal 1 (PTM1) to establish optimal $\mathrm{pH}$ and temperature set points (data not presented). At that time, higher rOvIFN- $\tau$ yields were obtained by replacing BSM with FM22 and PTM1 with PTM4, and by reducing the amount of PTM4 from 12 to $2 \mathrm{ml}^{-1}$. These improvements suggested that that the medium formulation could be optimized to improve yield. The FM22 growth medium was thus reformulated to (in grams per liter) $\mathrm{KH}_{2} \mathrm{PO}_{4}$, 25.7; $\left(\mathrm{NH}_{4}\right) 2 \mathrm{SO}_{4}, 5 ; \mathrm{K}_{2} \mathrm{SO}_{4}, 8.6 ; \mathrm{MgSO}_{4} \cdot 7 \mathrm{H}_{2} \mathrm{O}, 16.4$; and $\mathrm{CaSO}_{4} \cdot 2 \mathrm{H}_{2} \mathrm{O}, 1.4$, and sodium citrate was added at a final concentration of $20 \mathrm{mM}$ to reduce metallophosphate precipitates. PTM4 salt levels were lowered to $1.2 \mathrm{ml} \mathrm{l}^{-1}$ bolus from $2 \mathrm{ml} \mathrm{l}^{-1}$ into the vessel and as a supplement into the

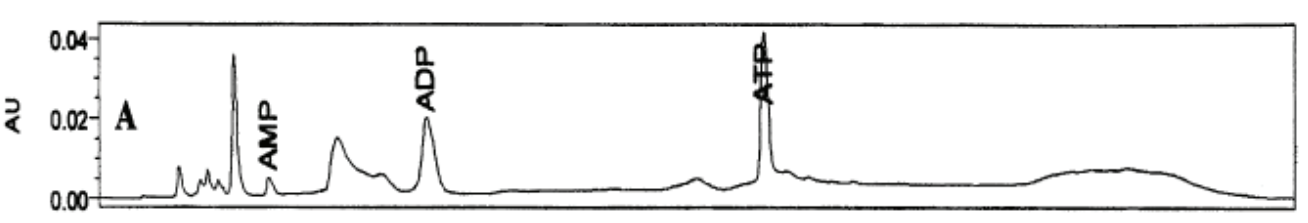

Figure 6. HPLC profiles of adenylate metabolic pools during expression of $\beta$-galactosidase. Each injection represents the extractable contents from 3.2 ODU of cell mass. The samples were obtained at 1.5 (a), 8 (b), 12 (c), 25 (d), 48 (e), 60 (f), and $72 \mathrm{~h}$ (g) after the transition from glycerol to methanol.
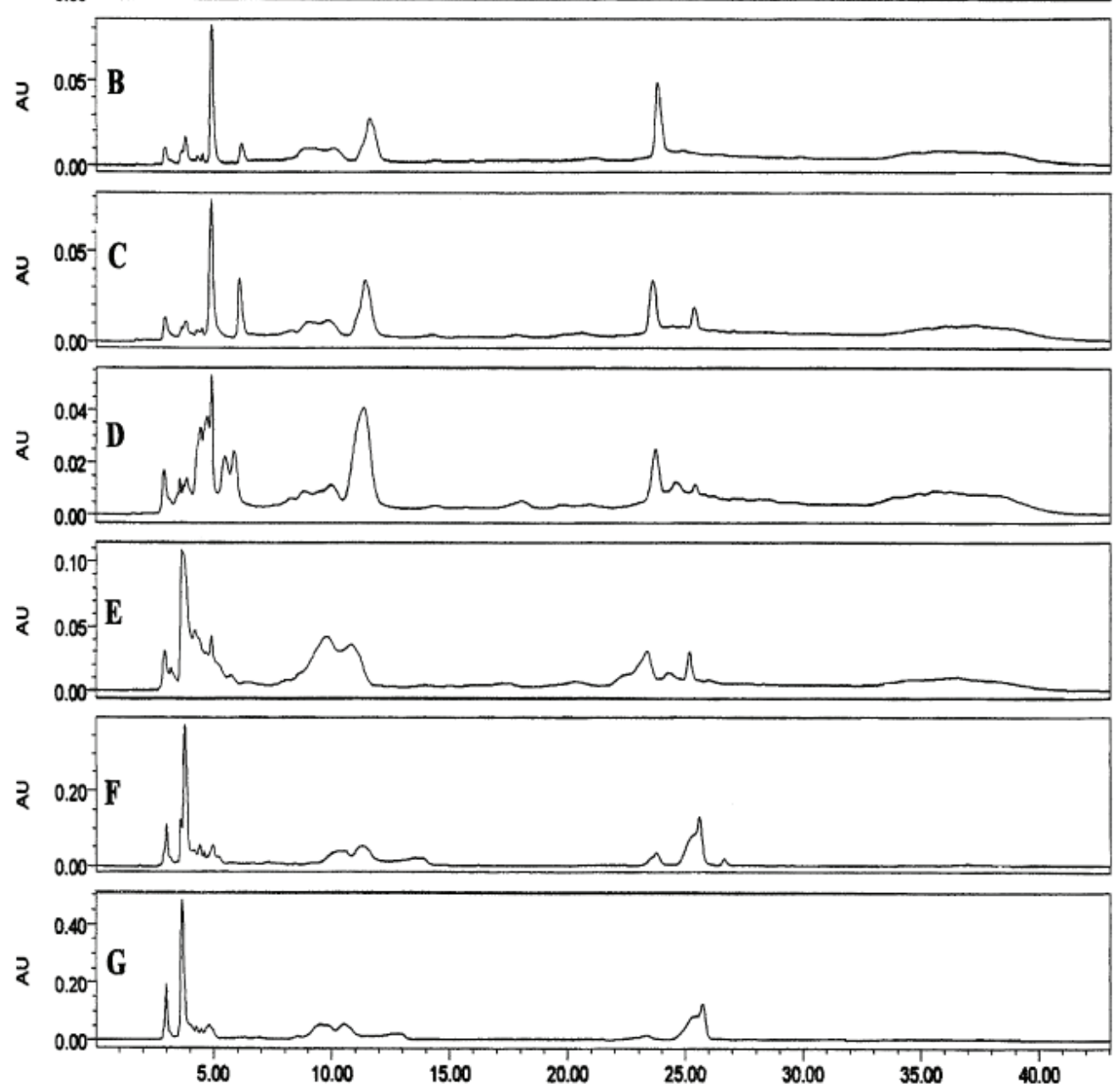

Minutes 
methanol. For the purpose of reducing metallophosphate precipitates, the salt solution was prepared in $20 \mathrm{mM}$ sodium citrate. With the reformulated medium, the solution remained clear at a $\mathrm{pH}$ of 6.5 , a value above the expected maximum process $\mathrm{pH}$. A growth experiment using citrate as the sole carbon source verified that citrate could not be utilized as a carbon source.

\section{Optimized process}

The fermentation for rOvIFN- $\tau$ was redesigned with the reformulated medium so that the maximum vessel capacity for cell mass and volume $\left(X_{\max } V_{\max }\right)$ would be reached approximately $37 \mathrm{~h}$ after induction of the AOX1 promoter. Three fermentations were then extended beyond this time to evaluate whether the optimized process conditions affected the onset of the decoupling point. A mean value for $\mu$ of $0.022 \pm 0.004$ per hour was maintained throughout the entire methanol feed profile. Moreover, two of the three fermentations maintained a positive steady-state production rate through to harvest. The process that decoupled did so after $44 \mathrm{~h}$ into the methanol feed profile. When the yield data were plotted against changing culture density normalized to growth rate, as described above (data not shown), a linear relationship with a constant productivity rate of $0.023 \pm 0.006 \mu \mathrm{g} \mathrm{g}^{-1} \mathrm{WCW} \mathrm{h}^{-1}\left(R^{2}=0.89\right)$ was obtained.

The $t$ test was performed to determine whether a significant difference existed between the parameters for the baseline and optimized processes (Table 1). Although product yields (on a per-liter basis) increased from $203 \pm 138$ to $337 \pm 79 \mathrm{mg} \mathrm{l}^{-1}$, there was no significant difference at $\alpha$ $=0.05$. This lack of significance can be attributed to high variability of the baseline runs. In addition, there was no significant difference in the rate of productivity $(p=0.764$, $\alpha=0.05$ ). However, total yields increased significantly from $559 \pm 262$ to $1404 \pm 305 \mathrm{mg}$, while variability between runs decreased, as indicated by the SD values for the critical control points.

Table 1. Comparison of optimized process to the baseline

\begin{tabular}{llll}
\hline & $\begin{array}{l}\text { Baseline } \\
\mathrm{SD}^{\mathrm{a}}\end{array}$ & $\begin{array}{l}\text { Optimized } \pm \\
\mathrm{SD}^{\mathrm{b}}\end{array}$ & $\begin{array}{c}p \\
\text { value }^{\mathrm{c}}\end{array}$ \\
\hline $\begin{array}{l}\text { Rate of product formation } \\
(\mathrm{mg} \text { rOvIFN- } \tau \text { g }\end{array}$ & $0.025 \pm 0.014$ & $0.022 \pm 0.004$ & 0.764 \\
$\begin{array}{l}\mathrm{WCW}^{-1} \mathrm{~h}^{-1} \text { ) } \\
\text { Maximum yield rOvIFN- } \tau \\
\left(\mathrm{mg} \mathrm{l}^{-1}\right)\end{array}$ & $203 \pm 138$ & $337 \pm 79$ & 0.120 \\
$\begin{array}{l}\text { Time on methanol prior to } \\
\text { decoupling (h) }\end{array}$ & $38.5 \pm 6.2$ & $46.8 \pm 2.4$ & 0.092 \\
$\begin{array}{l}\text { Total vessel production } \\
\text { (mg rOvIFN- } \tau)\end{array}$ & $557 \pm 357$ & $1,172 \pm 388$ & 0.011 \\
\hline
\end{tabular}

\footnotetext{
${ }^{a}$ Baseline data from the five runs also listed in Table 1

${ }^{\mathrm{b}}$ Medium used is $0.6 \times \mathrm{FM} 22$ but with $1.4 \times$ magnesium and calcium. The PTM4 salts were also reduced to $0.6 \times$ of the baseline ${ }^{\mathrm{c}}$ The $t$ test was used to determine if the difference was significant
}

\section{Discussion}

The prevalent paradigm for $P$. pastoris host systems is that recombinant proteins will be produced as long as the AOX1 promoter remains derepressed. The fermentation strategy typically used for these systems favors a long production phase because product yields are expected to accumulate throughout this period (Cregg et al. 1987; Byrne et al. 2000; Files et al. 2001). For some recombinant proteins, proteolytic degradation may be problematic. If so, this activity was considered all or nothing in that the recombinant protein was either susceptible to endogenous proteases or it was not. Should this condition exist, the use of protease inhibitors or protease-deficient strains might alleviate the problem, and extended production phases are again favored (Gleeson et al. 1998; Li et al. 2001). Yet, the yield profile of rOvIFN- $\tau$ did not fit these existing precepts. During the initial production phase, product did accumulate as predicted by a growth-associated model (Zhang et al. 2000b), but yields dropped dramatically at an average time of 38.5 $\pm 6.7 \mathrm{~h}$. Because product formation by a biological system is the outcome of many coupled enzymatic steps, the point at which production shifted from product accumulation to loss was designated as the decoupling point.

An unstructured model allows for greater process control because mean values and acceptable ranges for the state variables, culture growth, and product yield can be assigned according to the model. Process values can then be compared for acceptance. Underlying the growth associated model is the assumption that $\rho$ will remain constant as long as culture growth remains as quasi steady state (Zhang et al. 2000b). The decoupling point indicates that a shift from steady state occurred for both the rOvIFN- $\tau$ and the $\beta$-galactosidase production. In addition, stability of the rOvIFN- $\tau$ baseline fermentations was exacerbated by high run-to-run variability for the product yield parameters, although the culture growth rate parameters were highly reproducible (Figures 1 and 2). Because the 30-to 54-h time range represented one doubling of the culture density, and $\rho$ ranged between 0.015 and $0.047 \mathrm{mg} \mathrm{g}^{-1} \mathrm{WCW} \mathrm{h}^{-1}$, assigning a stop time simply based on a shortened production time would still have resulted in a highly variable process in terms of product yield and predicting the appropriate stop time.

Studies have shown that the energy charge of a cell is affected by recombinant protein expression. Katakura et al. (1998) reported that the energy charge of $P$. pastoris decreases during the production of recombinant human $\beta 2$-glycoprotein I domain V. Similarly, Trentmann et al. (2004) showed that limiting $\mu$ by limiting oxygen, which alters carbon flux rates, improves the stability of the $E$. coli fimbriae antibody fragment expressed in $P$. pastoris. These reports suggest that methanol cannot support the carbon and energy demands to sustain both growth and recombinant protein production. When the energy state of $P$. pastoris was evaluated during the production of recombinant 
$\beta$-galactosidase in our laboratory, the AEC dropped significantly between 12 and $25 \mathrm{~h}$ after adapting the culture to methanol. Although the production rate of $\beta$-galactosidase into the cytosol was not modeled (Figure 4), the pattern for product yield closely followed that for rOvIFN- $\tau$ (Figures $1 \& 2$ ). A similar production pattern was observed for the intracellular production of the heavy chain fragment of the botulinum neurotoxin fragment $\mathrm{C}$ (Bot C) (Zhang et al. 2000b). In that study, product accumulated in the cytosol for the first $12 \mathrm{~h}$ following induction. The major difference between the Bot $C$ and $\beta$-galactosidase yield curves was that the former material did not degrade. In combination, these studies suggest that a regulatory mechanism other than repression/derepression of the AOX1 promoter by methanol. If so, the cell must sense a change in its metabolic state and either down-regulate recombinant protein synthesis downstream from transcription or up-regulate proteolytic pathways. The latter event is supported by Sinha et al. (2004), who demonstrated that protease levels increased late in the production phase. Down-regulation of recombinant proteins has not been tested in P. pastoris, but work completed with the methylotrophic yeast $H$. polymorpha showed that the expression of E. coli $\beta$ galactosidase resulted in down-regulating the H. polymorpha AOX1 homolog methanol oxidase, and subsequently, $\beta$-galactosidase (Velkov et al. 1999).

Adenylate pools also directly influence protein synthesis and secretion. When the AEC in liver cells decreased from 0.90 to 0.80 , a half-maximal rate of $\mathrm{L}-\left[1-{ }^{14} \mathrm{C}\right]$ leucine incorporation into protein occurred, whereas an AEC drop of 0.85 from 0.90 resulted in protein secretion at half the maximal rate (Edwards et al. 1979). ATP is required for transporting secreted proteins from the reticulum system to the Golgi complex (Jamieson and Palade 1968). Depletion of ATP also leads to translational errors and the premature release of truncated proteins, which are then targeted for degradation by the ubiquitin pathway and the proteosome (Chuang et al. 2005). We have shown that a significant association exists between the decoupling point and a drop in the AEC (Figure 5). By using HPLC rather than enzymatic methods to quantify the AEC, these studies further demonstrate that the metabolic profiles of the cells shift significantly as production progresses (Figure 6). Comparison of our findings with the AEC studies reported by Edwards et al. (1979), Chuang et al. (2005), and Jamieson and Palade (1968) suggests that adenylates may either signal a shift in carbon flux or may directly regulate recombinant protein and/or protease synthesis.

The stability of the fermentation was also impacted by the availability of trace metals, which were adjusted by either changing their levels or by increasing their solubility by the addition of citrate. Our preliminary results agree with Brady et al. (2001), who reported higher yields of recombinant $\mathrm{P} 30 \mathrm{P} 2 \mathrm{mSP}_{19}$ with decreasing cation levels in the medium. Modifications to the basal salts and the levels of PTM4 salts resulted in a significant increase in pro- cess productivity on both a per-liter and, more significantly, a per-vessel basis. Moreover, run-to-run variability decreased due to the changes made to the trace metals, as evidenced by the lower CVs for $\rho(26 \%$ from $56 \%)$, yield on a per-liter basis (23-68\%), and for the predicted harvest time (decoupling) (5\% from 16\%). How the trace metals may be influencing metabolism and protein yield was not determined, but the data show that further process improvements may be gained by completing more thorough studies to optimize the trace metals and magnesium salts.

In conclusion, our work demonstrates the importance of understanding both the physiological state of the cell and the effects of growth parameters when developing P. pastoris mut $^{+}$fermentation processes. The quasi-steady-state model proposed for the Bot C (Zhang et al. 2000b) and the rOvIFN- $\tau$ production (Sinha et al. 2003) appeared correct in that no apparent shift in the growth rate occurred. However, our data indicate that a shift in cell physiology occurs, and the flow of carbon needed to produce recombinant protein then shifts to sustain growth. By understanding that the decoupling point is related to time on methanol rather than culture density, improvements to the rOvIFN- $\tau$ fermentation in terms of product yield and process stability was accomplished by extending the production period prior to the onset of the decoupling point.

Acknowledgements The funding for this research was generously supported by a grant provided by the Pepgen Corporation. We further acknowledge Dr. Gautam Sarath for data analysis, and the staff of the University of Nebraska Biological Process Development Facility.

\section{References}

Atkinson D, Walton G (1967) Adenosine triphosphate conservation in metabolic regulation: rate liver citrate cleavage enzyme. J Biol Chem 242:3239-3241

Babul J, Clifton D, Kretschmer M, Fraenkel D (1993) Glucose metabolism in Escherichia coli and the effect of increased amount of aldolase. Biochemistry 32:4685-4692

Brady C, Shimp R, Miles A, Whitmore M, Stowers A (2001) Highlevel production and purification of $\mathrm{P} 20 \mathrm{P} 2 \mathrm{MSP} 1_{19}$, an important vaccine antigen for malaria, expressed in the methylotrophic yeast Pichia pastoris. Protein Expr Purif 23:468-475

Byrne M, Titball R, Holley J, Smith L (2000) Fermentation, purification, and efficacy of a recombinant vaccine candidate against botulinum neurotoxin type F from Pichia pastoris. Protein Expr Purif 18:327-337

Chuang S-M, Chen L, Lambertson D, Anand M, Kinzy TG, Madura K (2005) Proteasome-mediated degradation of cotranslationally damaged proteins involves translation elongation factor $1 \mathrm{~A}$. Mol Cell Biol 25:403-413

Cregg JM, Barringer KJ, Hessler AY, Madden KR (1985) Pichia pastoris as a host system for transformations. Mol Cell Biol $5: 3376-3385$

Cregg JM, Tschopp J, Stillman C, Siegel R, Akong M, Craig W, Buckholz R, Madden K, Kellaris P, Davis G, Smiley B, Cruze J, Torregrossa R, Velice-lebi G, Thill G (1987) High-level ex- 
pression and efficient assembly of hepatitis B surface antigen in the methylotrophic yeast, Pichia pastoris. Bio/technology 5: 479-485

d'Anjou M, Daugulis J (2001) A rational approach to improving productivity in recombinant Pichia pastoris fermentation. Biotechnol Bioeng 72:1-11

Edwards K, Urban J, Schreiber G (1979) Relationship between protein synthesis and secretion in liver cells and the state of the adenine nucleotide system. Aust J Biol Sci 32:299-307

Files D, Ogawa M, Scaman C, Baldwin S (2001) A Pichia pastoris fermentation process for producing high-levels of recombinant human cystatin-C. Enzyme Microb Technol 29:335-340

Gleeson M, White C, Meininger D, Komives E (1998) Generation of protease-deficient strains and their use in heterologous protein expression. Methods Mol Biol 103:81-94

Jamieson J, Palade G (1968) Intracellular transport of secretory proteins in the pancreatic exocrine cell IV: metabolic requirements. J Cell Biol 39:589-603

Katakura Y, Zhang W, Guoqiang O, Takeshi K, Kishimoto M, Goto Y, Suga K-I (1998) Effect of methanol concentration on the production of human $\beta 2$-glycoprotein I domain $\mathrm{V}$ by a recombinant Pichia pastoris: a simple system for the control of methanol concentration using a semiconductor gas sensor. J Ferment Bioeng 86:482-487

Kobayashi K, Kuwae S, Ohya T, Ohda T, Ohyama M, Tomomitsu K (2000) High level secretion of recombinant human serum albumin by fed-batch fermentation of the methylotrophic yeast, Pichia pastoris, based on optimal methanol feeding strategy. J Biosci Bioeng 90:280-288

Li Z, Xiong F, Lin Q, d'Anjou M, Daugulis AJ, Yang DS, Hew CL (2001) Low-temperature increases the yield of biologically active herring antifreeze protein in Pichia pastoris. Protein Expr Purif 21:438-445

Lin-Cereghino J, Geoff P, Ilgen C, Cregg JM (2002) Production of recombinant proteins in ferment or cultures of the yeast Pichia pastoris. Curr Opin Biotechnol 13:329-332

Miller J (1992) A short course in bacterial genetics: a laboratory manual and handbook for Escherichia coli and related bacteria. Cold Spring Harbor Laboratory, Woodbury, pp 72-74

Plantz B, Andersen J, Smith L, Meagher M, Schlegel V (2003) Detection of non-host viable contaminants in Pichia pastoris cultures and fermentation broths. J Ind Microbiol Biotech 30:643-650

Sinha J, Plantz B, Zhang W, Gouthro M, Schlegel V, Liu C-P, Meagher M (2003) Improved production of recombinant ovine in- terferon- $\tau$ by mut ${ }^{+}$strain of Pichia pastoris using an optimized methanol feed profile. Biotechnol Prog 19:794-802

Sinha J, Plantz B, Inan M, Meagher M (2004) Causes of proteolytic degradation of secreted recombinant proteins production in methylotrophic yeast Pichia pastoris - case study with recombinant ovine interferon- $\tau$. Biotechnol Bioeng 89:102-112

Stratton J, Chiruvolu V, Meagher M (1998) High cell-density fermentation. In: Higgins DR, Cregg JM (eds) Methods in molecular biology: Pichia protocols. Humana, Totowa, pp 107- 120

Trentmann O, Khatri NK, Hoffmann F (2004) Reduced oxygen supply increases process stability and product yield with recombinant Pichia pastoris. Biotechnol Prog 20:1766-1775

Tschopp JF, Brust PF, Cregg JM, Stillman CA, Gingeras TR (1987) Expression of the lacZ gene from two methanol-regulated promoters in Pichia pastoris. Nucleic Acids Res 15:3859-3867

US Food and Drug Administration (2005) Code of federal regulations. General biological product standards, title 21, parts 610.9 and 610.13. US Government Printing Office, Washington, DC

Van Heeke G, Ott TL, Strauss A, Ammaturo D, Bazer FW (1996) High yield expression and secretion of the ovine pregnancy recognition hormone interferon-tau by Pichia pastoris.J Interferon Cytokine Res 16:119-126

Velkov VV, Matys VY, Sokolov DM (1999) How overproduction of foreign proteins affects physiology of the recombinant strains of Hansenula polymorpha. J Biosci 24:279-286

Wood T, Peretti S (1990) Depression of protein synthetic capacity due to cloned-gene expression in E. coli. Biotechnol Bioeng 36:865-877

Zhang W, Inan M, Meagher M (2000a) Fermentation strategies for recombinant protein expression in the methylotrophic yeast $P i$ chia pastoris. Biotechnol Bioprocess Eng 5:275-287

Zhang W, Bevins M, Plantz B, Smith L, Meagher M (2000b) Modeling Pichia pastoris growth on methanol and optimizing the production of a recombinant protein, the heavy-chain fragment $\mathrm{C}$ of botulinum neurotoxin, serotype A. Biotechnol Bioeng 70:1-8

Zhang W, Smith L, Plantz B, Schlegel V, Meagher (2002) Design of methanol feed control in Pichia pastoris fermentations based upon a growth model. Biotechnol Prog 18:1392-1399

Zhang W, Hywood Potter KJ, Plantz BA, Schlegel VL, Smith LA, Meagher MM (2003) Pichia pastoris fermentation with mixedfeeds of glycerol and methanol: growth kinetics and production improvement. J Ind Microbiol Biotechnol 30:210-215 